\title{
EFFICIENT EXTRACTION OF THIN FILM THERMAL PROPERTIES VIA PARAMETRIC MODEL ORDER REDUCTION AND OPTIMIZATION
}

\author{
T. Bechtold ${ }^{1}$, D. Hohlfeld ${ }^{2}$, E. B. Rudnyi ${ }^{3}$ \\ ${ }^{1}$ MCRTN COMSON, University of Wuppertal, Gauss Str. 20, 42119 Wuppertal, Germany \\ ${ }^{2}$ IMEC/Holst Centre, High Tech Campus 31, 5656 AE Eindhoven, The Netherlands \\ ${ }^{3}$ CADFEM GmbH, Marktplatz 2, 85567 Grafing b. München, Germany
}

\begin{abstract}
In this paper we present a novel highly efficient approach to determine material properties from measurement results. We apply our method to thermal properties of thin-film multilayers with three different materials, amorphous silicon, silicon-nitride and silicon-oxide. The individual material properties are identified by solving an optimization problem. For this purpose, we build a parameterized reduced-order model from a finite element (FE) model and fit it to the measurement results. The use of parameterized reduced order models within the optimization iterations speeds up the transient solution time by several orders of magnitude, while retaining almost the same precision as the full-scale FE model.
\end{abstract}

\section{KEYWORDS:}

Parametric model order reduction, thin film thermal properties, optimization

\section{INTRODUCTION}

The design of new microelectromechanical systems (MEMS) requires knowledge of properties of the used materials. This information is well known for most bulk materials. Monocrystaline silicon is the dominant material for MEMS fabrication and has been extensively studied so that its mechanical, thermal and electrical characteristics are precisely known [1]. Devices which are purely made from silicon, e.g. high frequency resonators or oscillating mirrors, can be exactly modeled due to the well known mechanical properties of the material. However, the fabrication of most MEMS devices involves the deposition of thin films, which are employed to fulfill specific functions, like sensing, actuation, passivation, etc.

Unfortunately, the material properties of thin films depend strongly on the fabrication parameters and subsequent process steps to the MEMS structure. In order to build an accurate MEMS model, the material properties of thin films have to be determined. Commercial MEMS foundries often supply the designer with a set of material properties; mostly covering mechanical and electrical properties.

In case of electro-thermal MEMS, the material thermal properties of thin film materials are of special interest. Often a good thermal isolation should be achieved between a sensing area and the supporting silicon frame (gas or infrared sensors, micro-filament). This is achieved by fabricating the thermally active component on a membrane, which is suspended on the silicon substrate. The transient characteristic of an electro-thermal MEMS are determined by thermal conductivity and specific heat of the employed materials. Together, these two parameters determine how fast and to what extent e.g. the temperature changes in response to applied amount of heat.

A conventional way to determine unknown thermal properties is to design, fabricate and characterize dedicated test structures, which employ the thin film material as a functional component. Simple test structure might include membranes [2] or beams [3] fabricated from a single material. These test structures feature a preferably simple geometry so that they can be described by analytical models. Mostly, the geometry is chosen in such a way as to result in a heat flow only in one direction, e.g. along a beam. In these cases one applies one dimensional model, which can be analytically solved. If the heat source and temperature distribution are measured on such test structures, one can determine the material thermal properties of the employed thin films.

Obviously, this approach requires a trade-off between modeling accuracy and fabrication requirements. Simple analytical models assume that heat flows only in one direction from source to sink. However, heat conduction is a distributed phenomenon and thus some fractions of the heat flow are neglected. Electrical connections to the thermally isolated heat source might be a cause of parasitic thermal flow. Therefore, the more precise numerical models are required for higher accuracy. However, the resulting equations can only be solved numerically at high computational effort. Furthermore, it is necessary to repeatedly adjust the model parameters in order to match the simulation and characterization results. This is usually performed within a time consuming optimization process, which requires time integration of the full-scale numerical model in each iteration [4].

In this work, we propose to use advanced mathematical algorithms of parametric model order reduction (pMOR) [5] to speed up the optimization of the numerical model and hence, enable accurate and efficient determination of the material thermal parameters of thin films. 
MEMS CASE STUDY - THERMALLY TUN-

\section{ABLE OPTICAL FILTER}

As a micromachined testcase we have chosen a thermally tunable optical filter [6] which features a multilayer square membrane with $550 \mu \mathrm{m}$ lateral dimensions, suspended over a silicon substrate (see Figure 1).

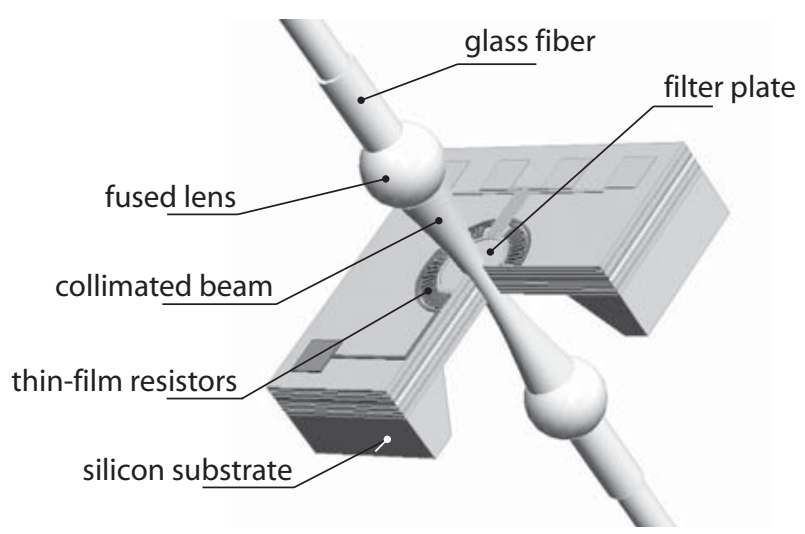

Figure 1 Membrane composed of multiple dielectric films with microstructured thin film resistors for heating and temperature sensing.

Three different multilayer designs were investigated, with multiple individual layers fabricated from amorphous silicon (a-Si), silicon nitride ( $\mathrm{SiN}$ ) and silicon oxide ( $\mathrm{SiO}$ ) by using plasma enhanced chemical vapor deposition. Figure 2 displays a schematic of the optical filter with thicknesses of respective layers. Design A employs 20 layer pairs of $\mathrm{SiO} / \mathrm{SiN}$, design $\mathrm{B}$ features 8 pairs of a-Si / $\mathrm{SiO}$ and design $\mathrm{C}$ is made from 10 pairs of a-Si / SiN. For all designs the cavity consists of amorphous silicon. The high number of layers is required to yield a good optical performance of the thin film interference filter. A meander shaped metal thin film heater is fabricated on top of the membrane.
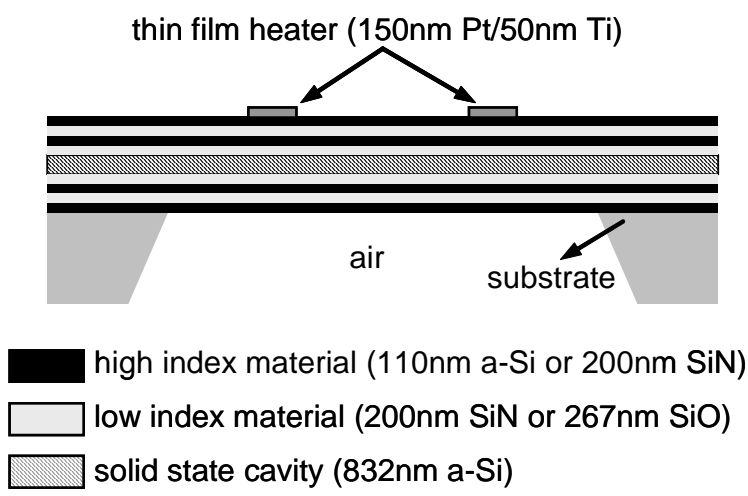

Figure 2 General structure of the optical filter stack, which consists of a cavity and two symmetrical dielectric Bragg reflectors (DBR). The number of material pairs with low and high refractive indexes in each $D B R$ varies depending on design.
The transient thermal response of the membrane is characterized by applying a rectangular voltage signal to the heating resistor so that a power of $1 \mathrm{~mW}$ is dissipated. The response of the membrane, as measured by the temperature sensor, is presented in Figure 3. After applying the heating

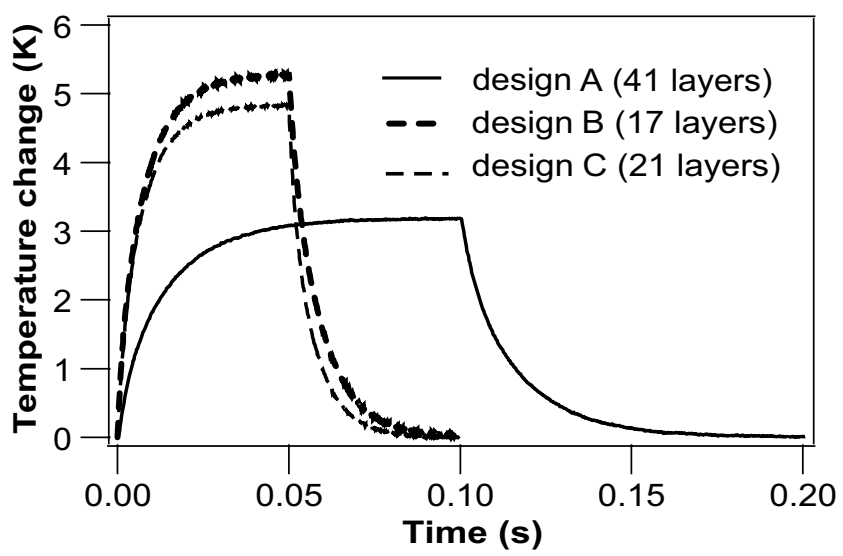

Figure 3 Temperature modulation of three multilayer membranes. A square wave heating power with a frequency of 10 resp. $5 \mathrm{~Hz}$ was applied to the membrane.

power, the temperature in the centre of the membrane increases until a maximum (steady-state) value is reached. Note, that design A shows lower steady state temperature and slower response than $\mathrm{B}$ and $\mathrm{C}$ as it features a much thicker membrane with a higher thermal conductance and higher thermal inertia. When no power is applied to the heater, the stored heat is dissipated to the surrounding media by conduction, free convection and radiation. Thus, the temperature decreases exponentially to its initial value.

We use full three-dimensional model with 60.000 nodes, which considers the lateral heat conduction through the membrane and the vertical heat conduction through the air beneath the membrane. The estimated Rayleigh number is about five, which indicates that heat convection above and below the membrane can be neglected. Radiation effects can also be neglected, as we obtain the measurement results at temperature changes of only a few degrees [7].

\section{PARAMETRIC MODEL ORDER REDUCTION}

Mathematical methods of model order reduction (MOR) enable a formal transformation of the physical model, that is, governing partial differential equation to a low-dimensional ordinary differential equation (ODE) system, as shown in Figure 4.

The intermediate level is a device level, that is, a high dimensional ODE system. The first conversion of the physical to the device model we perform via the finite element discretisation. The heat transfer within a hotplate is described through the following equations: 


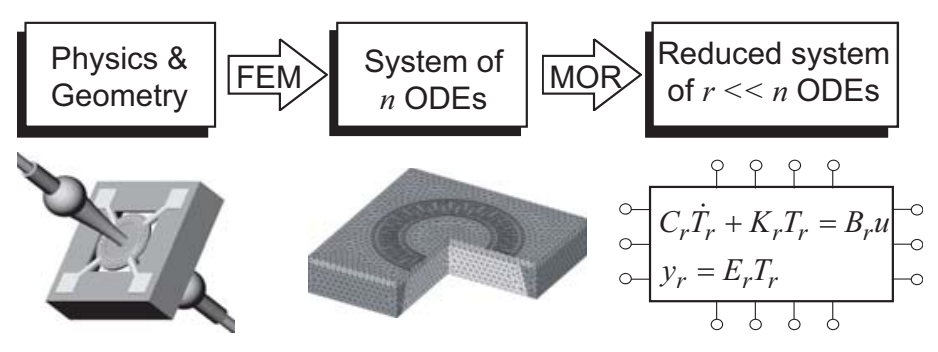

physical level

device level

system level

Figure 4 Place of model order reduction within a conversion process from physical to compact model.

$$
\nabla \bullet(\kappa \nabla T)+Q-\rho c_{p} \cdot \frac{\partial T}{\partial t}=0, Q=j^{2} R(T)
$$

where $\kappa(r)$ is the thermal conductivity in $\mathrm{W} / \mathrm{m} / \mathrm{K}$ at the position $r, c_{p}(r)$ is the specific heat capacity in $\mathrm{J} / \mathrm{kg} / \mathrm{K}$, $\rho(r)$ is the mass density in $\mathrm{kg} / \mathrm{m}^{3}, T(r, t)$ is the temperature distribution and $Q(r, t)$ is the heat generation rate per unit volume in $\mathrm{W} / \mathrm{m}^{3}$. We solve (1) with the initial condition $T_{0}=0$ and the Dirichlet boundary condition $T=0$ at the bottom of computational domain. Assuming that the heat generation is uniformly distributed within the heater and that the system matrices can be considered as temperature independent at the operation temperature, the finite element based spatial discretization of (1) leads to a large linear ODE system of the form:

$$
C \cdot \dot{T}+K \cdot T=B \cdot \frac{U^{2}(t)}{R(T)}, y=E \cdot T
$$

where $C$ and $K$ are the global heat capacity and heat conductivity matrices, $B$ is the load vector (matrix) and $E$ is the output vector (matrix). Equation (2) is a starting point for model order reduction, which leads to a system of the same form but with much smaller dimension. In the engineering problems, the most often used MOR approach is a moment matching approach [8], which is based on developing a transfer function

$$
H(s)=E(s C+K)^{-1} B
$$

of (2) into Taylor series around some value of the Laplace variable $s=s_{0}$, and then finding a much lower order $r$ system whose transfer function $H_{r}(s)$ has the same Taylor coefficients as $H(s)$, up to the degree $r$.

Note that in its original form model reduction does not allow to preserve parameters within the system matrices, which arise naturally in many applications, in the form of e.g. boundary conditions, material parameters or geometry parameters of the device. For microhotplate, (2) can be rewritten as:

$$
\left(C_{0}+\rho c_{p} \cdot C_{1}\right) \dot{T}+\left(K_{0}+\kappa \cdot K_{1}\right) T=b \frac{U^{2}(t)}{R(T)}, y=E \cdot T
$$

where the volumetric heat capacity $c_{p} \cdot \rho$ and thermal conductivity $\kappa$ are chosen as parameters and the system matrices are factorized. From numerical point of view, each parameter $p_{i}$ in the transfer function:

$$
H\left(s, p_{i}\right)=E\left[s\left(C_{0}+\rho c_{p} C_{1}\right)+K_{0}+\kappa K_{1}\right]^{-1} B
$$

of (4) is "equivalent" to the Laplace variable $s$. Hence, it is possible to generalize the moment matching approach and make a multivariate expansion of (5) with respect to the Laplace variable $s$ and parameters $p_{i}$. Thus the parameters are preserved in the symbolic form.

We have used the multivariate-moment-matching approach from [9] and were able to reduce the time for transient integration of the FE model by a factor of 330 . Parametric reduced models have only 60 degrees of freedom (DOF) but still provide high accuracy (see Figure 5).

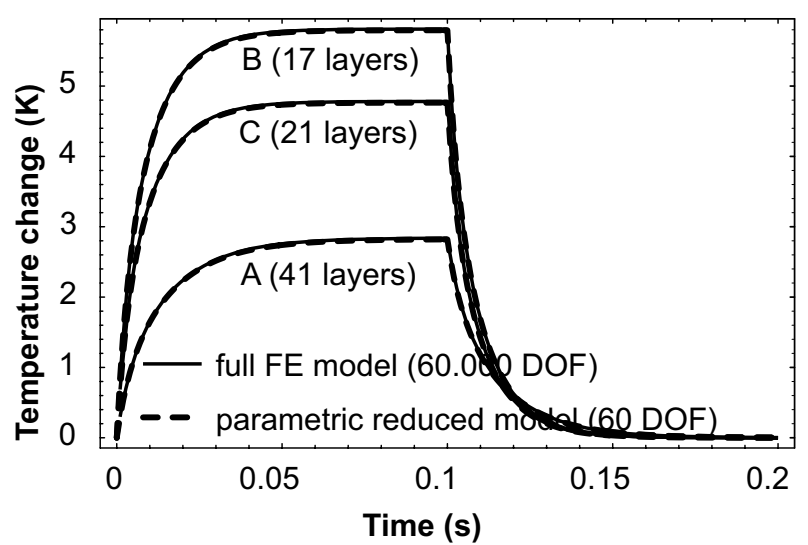

Figure 5 Comparison between the full FE models with 60.000 DOF and the parametric reduced models with 60 DOF for the initial parameters with $1 \mathrm{~mW}$ heating power (linear model is applicable for small heating power levels only).

\section{OPTIMIZATION RESULTS}

Because material parameters are preserved as parameters within the reduced model, they can be altered in each iteration of the optimization process. By defining an objective function, which characterizes the difference between simulated and measured results, data fitting cycle according to the algorithm in Figure 6 is performed. Note that, thanks to pMOR, no new FE model must be built and reduced in each iteration, as done in [10].

We consider the multilayer membrane as a solid volume i.e. the membrane consists of a single material with 'effective' properties. These are calculated by averaging the material characteristics over the membrane's cross-section and are used as parameters for the optimization procedure. Figure 7 shows the convergence of thermal conductivity during the course of optimization. As we investigated three different multilayer designs, it was possible to recover the individual material properties of all three thin film materials. The estimated values are displayed in Table 1. 


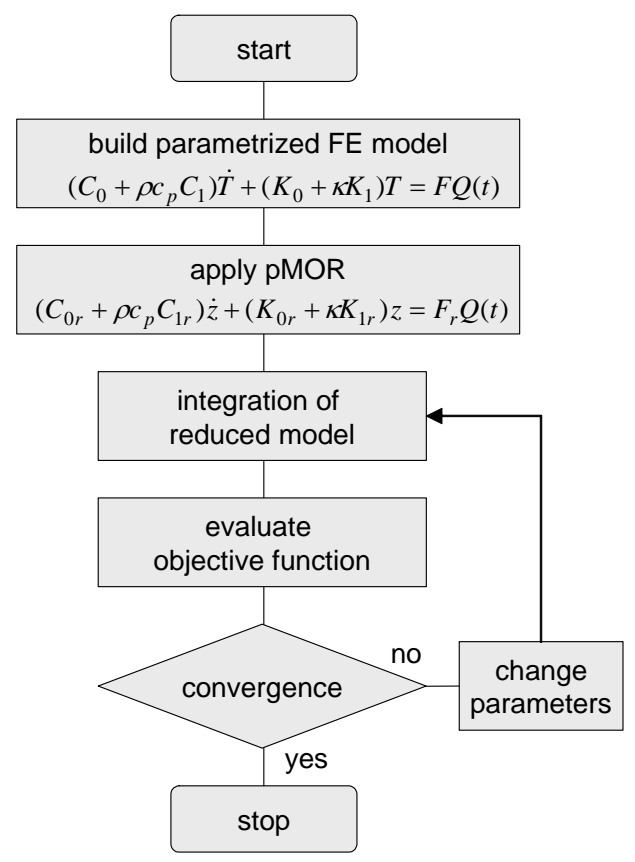

Figure 6 Algorithm for fast determination of material properties via parametric model order reduction.

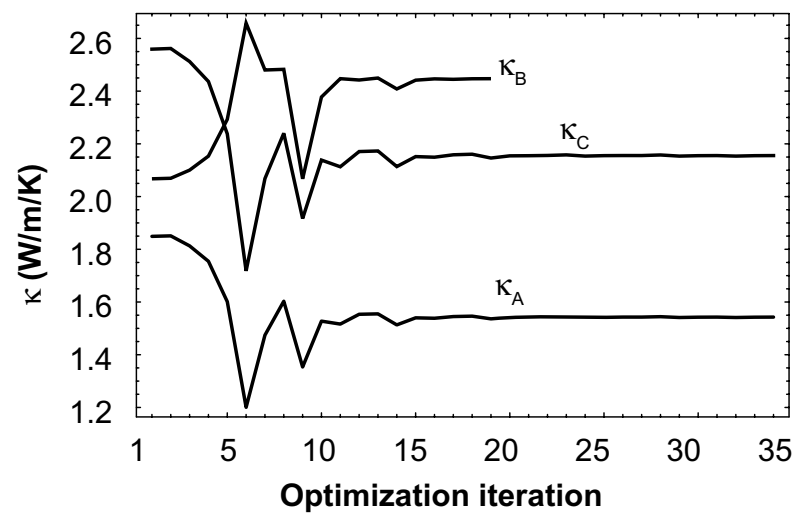

Figure 7 Convergence of thermal conductivity of the membrane volume during the course of optimization.

Note that it is not possible to independently determine the specific heat and mass density, as only their product is present in the system's heat capacity matrix. Figure 8 shows the comparison between the measured temperature response, the initial FE model and the model with optimized parameters.

Table 1 Determined thermal material properties for amorphous silicon, silicon nitride and silicon dioxide.

\begin{tabular}{c|ccc}
\hline & $\begin{array}{c}\mathbf{a}-\mathbf{S i} \\
(\mathbf{1 1 0 n m})\end{array}$ & $\begin{array}{c}\text { SiN } \\
(\mathbf{2 0 0 n m})\end{array}$ & $\begin{array}{c}\text { SiO } \\
\mathbf{( 2 6 7 n m )}\end{array}$ \\
\hline$\kappa(\mathrm{W} / \mathrm{m} / \mathrm{K})$ & 3.39 & 0.96 & 1.69 \\
$\rho c_{p}\left(10^{6} \mathrm{~J} / \mathrm{m}^{3} / \mathrm{K}\right)$ & 1.30 & 1.93 & 1.68 \\
\hline
\end{tabular}

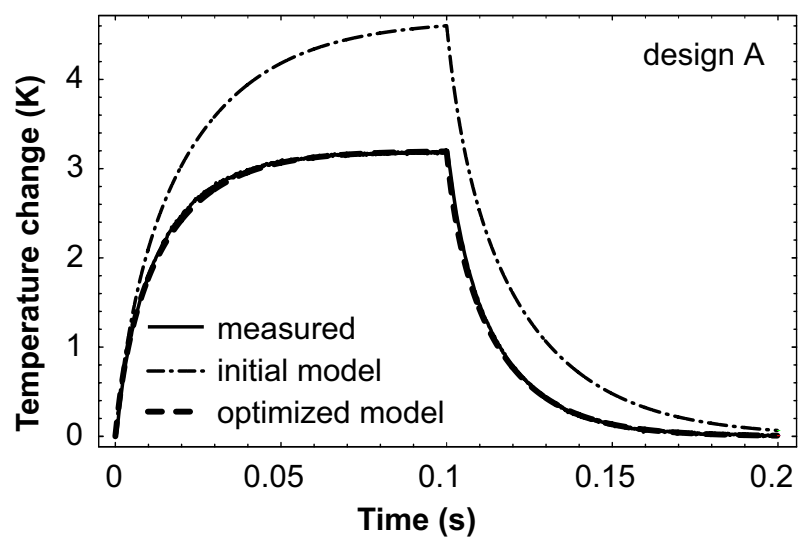

Figure 8 Measured and simulated transient curves after 35 optimization cycles with $1 \mathrm{~mW}$ heating power.

\section{CONCLUSION AND OUTLOOK}

We have demonstrated a methodology for the efficient determination of material properties via parametric model order reduction and subsequent automatic parameter optimization. We were able to extract thermal parameters of thin films from transient thermal characterization results, while using highly accurate three-dimensional numerical models of test structures.

The next step would be to include temperature dependence of the material parameters, which requires application of pMOR to non-linear systems [11] and also to include the radiation effects, which become significant at temperatures above $300^{\circ} \mathrm{C}$.

\section{REFERENCES}

[1] R. Hull (ed.), Properties of Crystalline Silicon, Institution of Engineering and Technology, (1999).

[2] F. Volklein, et al. Proc. Int. Conf. Thermoelectrics, pp. 711-718, (1997).

[3] A. Roncaglia, et al., Proc. TRANSDUCERS, pp. 615618, (2007).

[4] N. Stojanovic, et al., IEEE J. Microelectrom. Syst., 16, pp. 1269-1275, (2007).

[5] L. H. Feng, Math. Comp. Sim., 68(3), pp. 221-234, (2005).

[6] D. Hohlfeld, et al., IEEE J. Microelectrom. Syst., 16(3), pp. 500-510, (2007).

[7] L. La Spina, et al., IEEE J. Microelectrom. Syst., 16, pp. 675-683, (2007).

[8] R. W. Freund, J. Comp. Appl. Math., 123, pp. 395-421, (2000).

[9] D. Celo, et al., IEEE Trans. on Comp. and Pack. Techn., 28(1), pp. 70-79, (2005).

[10] J. S. Han, et al., J. Micromech. Microeng., 15(4), pp. 822-832, (2005).

[11]B. Bond, et al., IEEE Trans. on Comp.-Aided Design of Int. Circ. Syst., 26(12), (2007).

CONTACT: Tamara.Bechtold@math.uni-wuppertal.de 